\title{
Cirugía cardiaca con circulación extracorpórea: síndrome vasoplégico y vasopresina
}

\author{
MACARENA GILBERT ${ }^{a}$, GUILLERMO LEMA
}

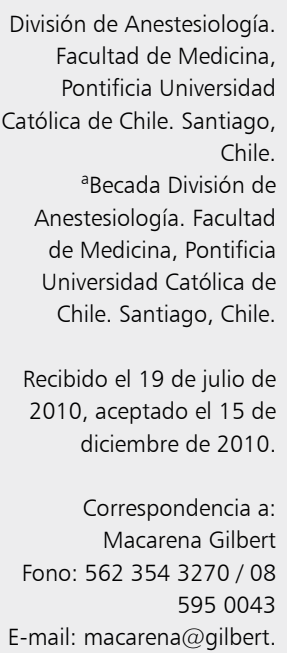

\section{Vasoplegic syndrome and its treatment with vasopressin during cardiac surgery with cardiopulmonary bypass}

Cardiac surgery with cardiopulmonary bypass is associated with systemic inflammatory response. In some cases this clinical condition is characterized by severe hypotension due to low systemic vascular resistance during and after cardiopulmonary bypass. A few of these cases do not respond to volume or catecholamines. This condition is known as vasoplegic syndrome. Its etiology is not fully understood today and carries associated morbidity and mortality. In this syndrome, vasopressin levels are reduced, as in septic and hypovolemic shock. Supplementary vasopressin improves blood pressure and might be considered as an alternative treatment. Several reports have shown benefits when used alone or in combination with catecholamines. However, further studies are necessary to find the most appropriate use of the drug for vasoplegic syndrome.

(Rev Med Chile 2011; 139: 368-372).

Key words: Cardiopulmonary bypass: Thoracic surgery; Vasoplegia; Vasopressins.
L a cirugía cardiaca con circulación extracorpórea (CEC) se acompaña de una respuesta inflamatoria sistémica, caracterizada por vasodilatación y por disminución de la resistencia vascular sistémica (RVS). Esta condición patológica se traduce en importante morbilidad y mortalidad ${ }^{1}$. El cuadro clínico se asocia a un gasto cardiaco (GC) elevado y evidencias de disfunción de órganos: oliguria, elevación de lactato isquemia coronaria, entre otros.

La etiología parece ser la activación de un sistema de mediadores neuroendocrinos e inmunológicos que generan injuria endotelial, liberación de citoquinas y otros mediadores de la inflamación. El mecanismo responsable se conoce sólo en forma parcial $^{2}$. Su tratamiento involucra la optimización en la administración de volumen y uso de vasopresores para restablecer la RVS y la presión arterial (PA) a valores normales. Desafortunadamente, los cuadros clínicos avanzados de vasodilatación se asocian a falta de respuesta adrenérgica, condición conocida como síndrome vasoplégico (SV). En $10 \%$ de los casos hay resistencia al uso de catecolaminas y efectos colaterales por las elevadas dosis de vasopresores utilizados ${ }^{3}$. El cuadro clínico se asocia con mayor frecuencia a períodos prolongados de CEC, situaciones de emergencia, uso prolongado de vasopresores y pacientes de edad avanzada. Si persiste, pese a un adecuado manejo farmacológico, se asocia a una elevada tasa de mortalidad ${ }^{4}$. Las diferentes series reportan incidencias variadas de SV, dependiendo de la definición exacta que los autores den al cuadro clínico descrito. Aun así, y dada la asociación a estadías prolongadas en UCI, períodos hospitalarios extendidos, así como mayores costos de hospitalización, constituye un tema relevante para todos aquellos encargados del manejo de estos pacientes. 
Síndrome vasoplégico y vasopresina - M. Gilbert et al

\section{Fisiopatología}

La PA normal es resultado de la interacción entre el sistema nervioso simpático, el sistema renina angiotensina (SRA) y el sistema argininavasopresina. El SV se caracteriza por una marcada hipotensión, como resultado de una vasodilatación periférica importante. El cuadro clínico descrito responde de muy mala manera al uso de vasopresores. La sepsis es la causa más frecuente de shock, y es en esta patología donde se concentra el mayor número de estudios; las extrapolaciones hacia otras formas de SV deben hacerse con cautela, ya que es probable que exista activación de otros mecanismos en las formas no infecciosas de shock.

En la cirugía cardiaca con CEC, la inflamación sistémica puede producir vasoplegia, hipoperfusión prolongada, disfunción multiorgánica y muerte. En todas las formas de shock se han consignado elevadas concentraciones plasmáticas de catecolaminas, así como activación del SRA ${ }^{5}$. Es esta observación la que hace planteable que el principal mecanismo responsable del SV sea la incapacidad del endotelio de vasocontraerse. Los mecanismos patogénicos implicados son muchos, entre ellos, la activación de canales de potasio sensibles a ATP (canales $\mathrm{K}_{\text {ATP }}$ ), la activación de la forma inducible de la óxido nítrico-sintetasa y deficiencias en los niveles de la hormona vasopresina (AVP). El resultado es un desbalance entre sistemas vasopresores y vasodilatadores, con una excesiva dilatación de los vasos de resistencia.

La vasoconstricción normal requiere que ligandos endógenos se unan y activen receptores de superficie de células de músculo liso vascular. Esta interacción, por medio de segundos mensajeros, aumenta la concentración citosólica de $\mathrm{Ca}^{++}$ produciendo contracción. Una vasoconstricción sostenida requiere de la entrada de $\mathrm{Ca}^{++}$desde el extracelular, por lo que la hiperpolarización de la membrana previene la vasoconstricción aun en presencia de ligandos vasoconstrictores. Existe una gran variedad de canales y transportadores iónicos, particularmente canales de $\mathrm{K}^{+}$, que contribuyen al potencial de membrana. La apertura de canales $\mathrm{K}_{\text {ATP }}$ permite la salida masiva de $\mathrm{K}^{+}$desde el intracelular, hiperpolarizando la membrana e impidiendo la entrada de $\mathrm{Ca}^{++}$al citosol. Estos canales son activados fisiológicamente por la disminución en la concentración celular de ATP y el aumento en las concentraciones celulares de $\mathrm{H}^{+} \mathrm{y}$ lactato. Todas las condiciones que comprometan la oxigenación tisular, con la consecuente acidosis láctica, probablemente activen canales $\mathrm{K}_{\mathrm{ATP}}$ en vasos, contribuyendo así al SV .

El aumento en la síntesis de óxido nítrico (NO) también puede contribuir a la hipotensión y resistencia a drogas vasopresoras. El NO, mediante la activación de la guanilato ciclasa, produce GMPc, otro vasodilatador endotelial. Tanto en shock séptico como en shock hemorrágico, la producción de NO está aumentada como resultado de una mayor expresión de la forma inducible de la óxido nítrico-sintetasa. Sin embargo, esto no se ha podido demostrar en el caso de la cirugía con $\mathrm{CEC}^{7}$. Es posible que vías independientes de $\mathrm{NO}$ sean de mayor relevancia para la activación de GMPc y el posterior desarrollo de SV.

Por último, como parte de una respuesta barorefleja normal, hay secreción de AVP, encargada de regular la permeabilidad de los túbulos colectores renales y conservar agua. Normalmente la AVP juega un rol menor en la regulación de la PA, pero en respuesta a la hipotensión de una sepsis o hemorragia importante, las concentraciones plasmáticas aumentan en forma muy marcada contribuyendo inicialmente, a la mantención de la $\mathrm{PA}^{8}$. En la medida que el shock empeora, estas elevadas concentraciones iniciales disminuyen y el efecto regulador de la presión recae en el resto de los mecanismos vasoconstrictores presentes ${ }^{9}$. No se conoce con certeza el mecanismo por el cual estos niveles disminuyen, pero pudiera corresponder a la depleción de las reservas neurohipofisiarias o a una inhibición de la producción, liberación, o ambos.

Hace más de un siglo atrás se caracterizó el efecto fisiológico de la vasopresina u hormona antidiurética por los investigadores Oliver y Schaefer, quienes observaron el efecto de extractos de glándula pituitaria sobre la $\mathrm{PA}^{10}$. Fue posteriormente aislada en 1951, para ganar popularidad en el manejo médico de várices esofágicas sangrantes y diabetes insípida ${ }^{11}$. Este nonapéptido es sintetizado en los núcleos supraópticos y paraventricular del hipotálamo, para ser posteriormente almacenado en el lóbulo posterior de la hipófisis y liberado a la circulación en presencia de estímulos osmóticos (hiperosmolaridad plasmática como principal estímulo) y no osmóticos (hipotensión, dolor, náuseas e hipoxia). Es sintetizado en menor grado por el corazón en respuesta a un aumento del 
estrés de la pared ventricular y por la glándula adrenal frente a un aumento en la secreción de catecolaminas ${ }^{12}$. Participa en múltiples otros procesos fisiológicos desde control de temperatura, liberación de insulina y corticotropina, memoria y comportamiento social ${ }^{13}$.

La AVP ejerce su efecto a través de los receptores de AVP y ocitocina. Los receptores de AVP corresponden a una superfamilia de receptores acoplados a proteína $G$, que se subdividen en tres: $\mathrm{V}_{1}$ o receptor vascular, $\mathrm{V}_{2}$ o receptor antidiurético y $V_{3}$ o receptor hipofisiario. $V_{1}$ se localiza en el músculo liso vascular y es responsable de mediar la vasoconstricción a través del aumento intracelular de $\mathrm{Ca}^{++}$mediante señalización de fosfolipasa C. $V_{2}$ se ubica a nivel de túbulos colectores renales y células endoteliales y media el efecto antidiurético a través de la activación de adenil ciclasa; $\mathrm{V}_{3}$ es el receptor hipofisiario responsable de la regulación central. La AVP posee escaso efecto sobre la PA en condiciones y concentraciones plasmáticas normales; sin embargo, junto con las catecolaminas, contribuye a mantenerla mediante receptores $V_{1}$ en estados hipovolémicos. A diferencia de las catecolaminas, la AVP es un potente vasoconstrictor de la piel, músculo esquelético, grasa, páncreas y glándula tiroides, no tanto así de la circulación coronaria y cerebral. Así mismo, no produce vasoconstricción mesentérica o pulmonar in vitro ${ }^{14}$. La AVP es también capaz de aumentar la sensibilidad del músculo liso vascular a otros agentes vasoconstrictores y bloquear canales $\mathrm{K}_{\mathrm{ATP}}$ de manera dosis dependiente. Esta acción podría ser responsable, en parte, de restablecer el tono vascular en los estados vasodilatados post CEC.

\section{Factores de riesgo}

Se han establecido diversos factores de riesgo independientes para SV en el período posterior a la CEC. En 1998, Argenziano y cols buscaron establecer en forma prospectiva la incidencia de SV y los factores predisponentes asociados. Estudiaron a 145 pacientes sometidos a cirugía cardiaca, dentro de los cuales reportó $8 \%$ de pacientes con signos de shock vasoplégico (definido para ese trabajo como presión arterial media $[\mathrm{PAM}]<70 \mathrm{mmHg}$, índice cardiaco $>2,5 \mathrm{lt} / \mathrm{min} . / \mathrm{m}^{2}$ y dependencia a norepinefrina) y establecieron como predictores independientes de SV una fracción de eyección
$<35 \%$ y el antecedente de uso preoperatorio de drogas del tipo IECA ${ }^{3}$. Mekontso-Dessap y cols, posteriormente ratifican algunos de estos hallazgos, estableciendo un riesgo relativo de 2,78 al antecedente de uso preoperatorio de drogas IECA, independiente de la función ventricular previa ${ }^{15}$. Otros factores de riesgo identificados han sido: infusiones preoperatorias de heparina, uso de antagonistas de canales de $\mathrm{Ca}^{++}$, condiciones de estrés elevado como el infarto, presencia de diabetes y el uso de protamina ${ }^{16}$.

\section{Evidencia}

Llama la atención que en pacientes sometidos a cirugía coronaria con buena evolución postoperatoria, hay aumentos de hasta 6 veces de los niveles basales de AVP en un período hasta $12 \mathrm{~h}$ posterior a la $\mathrm{CEC}^{14}$. Comparativamente, en presencia de SV post CEC se ha documentado que, aun habiendo niveles de AVP dentro de rangos para la osmoregulación de un sujeto normal, estos son menores a los niveles esperados para el grado de hipotensión presente. Recientemente, Jochberger y cols, evaluaron en forma prospectiva las concentraciones plasmáticas de AVP en pacientes con vasoplegia después de una cirugía cardiaca con CEC y los compararon con los niveles en pacientes sometidos a cirugía coronaria no complicada ${ }^{17}$. El alza en la concentración de AVP fue significativamente más bajo el primer día postoperatorio en el grupo vasoplégico, observándose una concentración significativamente mayor al grupo control sólo a partir del tercer día postoperatorio.

La corrección de las bajas concentraciones de AVP se correlaciona con un aumento significativo de la PA. Los primeros reportes del uso de AVP en el manejo de SV fueron esencialmente reportes de casos. El primer trabajo con grupos aleatorios que comparó AVP con suero fisiológico estudió 10 pacientes con criterios de SV posterior a la instalación de un dispositivo de asistencia ventricular. Ellos fueron distribuidos en forma aleatoria a recibir $\operatorname{AVP}(0,1 \mathrm{U} / \mathrm{min})$ o placebo. La AVP aumentó en forma significativa la PAM y la RVS en todos los pacientes, independiente de los niveles plasmáticos. Asimismo se observó una disminución en los requerimientos de norepinefrina, con lo cual los autores concluyeron que la AVP era un vasopresor efectivo para el tratamiento del SV en este tipo de 
cirugía $^{18}$. Dünser y cols posteriormente reportaron un estudio prospectivo, aleatorio y controlado de 48 pacientes con SV secundario a cirugía cardiaca o síndrome de respuesta inflamatoria sistémica (ambos con o sin sepsis) resistente al manejo con catecolaminas. Todos tenían PAM $<70 \mathrm{mmHg}$ a pesar de una adecuada reposición de volumen, con requerimientos de norepinefrina $>0,5 \mathrm{ug} / \mathrm{kg} /$ min. Los pacientes fueron distribuidas en forma aleatoria a recibir AVP $4 \mathrm{U} / \mathrm{hr}$ más norepinefrina para una PAM $>70 \mathrm{mmHg}$ o norepinefrina sola, encontrando que la PAM e índice cardiaco eran significativamente mayores en el grupo que recibió AVP más norepinefrina. Recientemente, 27 pacientes usuarios de IECA operados con CEC a recibir aleatoriamente en forma profiláctica AVP o solución salina 20 minutos antes del inicio de la CEC. Si bien no se encontraron cambios en cuanto a la PA previo al inicio de la CEC, fue evidente el menor tiempo de uso y dosis de norepinefrina con el que se debió apoyar a los pacientes que recibieron AVP, así como un menor número de episodios hipotensivos y menor tiempo de estadía en $\mathrm{UCI}^{19}$.

Esta y otras evidencias clínicas sugieren que el uso de AVP es una alternativa o una adyuvante importante al mejorar la PAM en pacientes con SV post CEC.

Es importante destacar que los distintos estudios clínicos rara vez utilizan AVP como vasoconstrictor de primera línea en el manejo post CEC. Las dosis utilizadas varían sustancialmente entre trabajos (entre 0,01-0,1 U/min), así como la duración de su infusión, con lo cual cualquier comparación se hace difícil. La mayoría de los ensayos relevantes que usan AVP lo hacen en asociación a otros vasopresores, en su mayoría agonistas adrenérgicos. En muchos de los casos se agrega AVP cuando las dosis de vasopresores (en su mayoría norepinefrina) son muy elevadas o la PAM ha disminuido considerablemente, probablemente porque estos últimos son habitualmente efectivos, predecibles, fácilmente titulables y conocidos por aquellos que los indican. Es por esto que el uso de AVP, hasta ahora, se ha centrado fundamentalmente en los estados vasodilatados en los cuales no hay respuesta a los esquemas habituales y como una droga de rescate; sólo algunos centros cardio-quirúrgicos la usan como droga de primera línea. Los escasos trabajos existentes utilizan esquemas muy variados, lo que dificulta la interpretación y comparación de sus resultados.
Si aceptamos que los niveles de AVP se encuentran disminuidos en SV, ¿cuál es el grado de deficiencia que realmente existe? y ¿cuál es la concentración a la cual debemos apuntar que ofrezca las mayores ventajas fisiológicas?

Al revisar la literatura médica se puede apreciar la falta de estudios enfocados a revisar el "outcome" de un escenario no tan infrecuente como es el SV post CEC. La evidencia disponible es muchas veces heterogénea; gran parte de la evidencia con respecto al uso de la AVP en humanos corresponde a reporte de casos, análisis retrospectivos o estudios comparativos pequeños. Asimismo, aún no se cuenta con estudios multicéntricos controlados $\mathrm{y}$ aleatorios que relacionen los efectos de AVP en cuanto a "outcome" global. Sin embargo, el uso de AVP en el contexto de un SV parece prometedor. La infusión continua en pacientes con hipotensión refractaria post CEC ha mostrado mejoras en los niveles de diuresis ${ }^{20}$. Por otro lado, la AVP también ha demostrado ser un potente vasoconstrictor esplácnico; se ha planteado la posibilidad de isquemia gastrointestinal en pacientes susceptibles ${ }^{21}$.

A la fecha, es probable que la AVP (junto a las ya establecidas epinefrina/norepinefrina) juegue un papel importante en la regulación de la PA en estados de vasodilatación. Aún queda por establecer si la AVP es un adyuvante de otras catecolaminas, si por si sola es igual, mejor o peor que la epinefrina/norepinefrina o, si en conjunto, adquieren características terapéuticas aún por definir en su totalidad.

\section{Referencias}

1. Dünser M, Mayr A, Ulmer H, Knotzer H, Sumann G, Pajk W, et al. Arginine Vasopressin in advanced vasodilatory shock. A prospective, randomized, controlled study. Circulation 2003; 107: 2313-9.

2. Taylor K. SIRS: the systemic inflammatory response syndrome after cardiac operations. Ann Thorac Surg 1996; 61: 1607-8.

3. Egi M, Bellomo R, Langenberg C, Haase M, Haase A, Pharm B, et al. Selecting a vasopressor drug for vasoplegic shock after adult cardiac surgery: A systematic literature review. Ann Thorac Surg 2007; 83: 715-23.

4. Gomes W, Carvalho A, Palma J, Teles CA, Branco JN, Silas MG, et al. Vasoplegic syndrome after open heart surgery. J Cardiovascular Surg 1998; 39: 619-23.

5. Benedict C, Rose J. Arterial norepinephrine changes in 
patients with septic shock. Circ Shock 1992; 38: 165-72.

6. Landry D, Oliver J. The pathogenesis of vasodilatory shock. N Engl J Med 2001; 345: 588-95.

7. Cumming A, Driedger A, McDonald J, Lindsay R, Solez $\mathrm{K}$, Linton AL. Vasoactive hormones in the renal response to systemic sepsis. Am J Kidney Dis 1988; 11: 23-32.

8. Den Ouden D, Meinders A. Vasopressin: physiology and clinical use in patients with vasodilatory shock: a review. The Netherlands Journal of Medicine 2005; 63: 4-13.

9. Morales D, Madigan J, Cullinane S, Heath $\mathrm{W}, \mathrm{Oz} \mathrm{W}$, Oliver JA, et al. Reversal by vasopressin of intractable hypotension in the late phase of hemorrhagic shock. Circulation 1999; 100: 226-9.

10. Vincent J, Su F. Physiology and pathophysiology of the vasopressinergic system. Best Practice \& Research Clinical Anaesthesiology 2008; 22: 243-52.

11. Overgaard C, Dzavik V. Inotropes and Vasopressors: Review of Physiology and Clinical Use in Cardiovascular Disease. Circulation 2008; 118: 1047-56.

12. Hupf H, Grimm D, Riegger GA, Schunkert H. Evidence for a vasopressin system in the rat heart. Circ Res 1999; 84: 365-70.

13. Guillon G, Grazzini E, Andrez M, Breton C, Trueba M, Serradeil-LeGal C, et al. Vasopressin: a potent autocrine/ paracrine regulator of mammal adrenal functions. Endocr Res 1998; 24: 703-10.

14. Booth J, Schinderle D, Welsby I. Pro: Vasopressin Is the Vasoconstrictor of Choice After Cardiopulmonary Bypass. Journal of Cardiothoracic and Vascular Anesthesia 2002; 16: 773-5.
15. Mekontso-Desap A, Houël R, Soustelle C, Kirsch M, Thebert D, Loissance D. Risk factors for post-cardiopulmonary bypass vasoplegia in patients with preserved left ventricular function. Ann Torac Surgery 2001; 71: 1428-32.

16. Shanmugam G. Vasoplegic syndrome. The role of methylene blue. European Journal of Cardio-thoracic Surgery 28; 2005: 705-10.

17. Jochberger S, Velik-Salchner C, Mayr V, Luckner G, Wenzel V, Falkensammer G, et al. The vasopressin and copeptin response in patients with vasodilatory shock after cardiac surgery: a prospective, controlled study. Intensive Care Med 2009; 35: 489-97.

18. Argenziano M, Choudhri A, Oz M, Rose E, Smith C, Ladry D. A prospective randomized trial of arginine vasopressin in the treatment of vasodilatory shock after left ventricular assist device placement. Circulation 1997; 96 (9 Suppl) 286-90.

19. Morales D, Garrido M, Madigan J, Helman D, Faber J, Williams $\mathrm{M}$, et al. A double-blind randomized trial: prophylactic vasopressin reduces hypotension after cardiopulmonary bypass. Ann Thorac Surg 2003; 75: 926-30.

20. Maserri P, Murphy S, Kouchoukos N. Vasopressin therapy for vasoplegic syndrome dollowing cardiopulmonary bypass. J Cardiac Surg 2002; 17: 485-9.

21. Nygren A, Thorén A, Ricksten S. Vasopressin decreases intestinal mucosal perfusion: a clinical study on cardiac surgery patients in vasodilatory shock. Acta Anaesthesiol Scand 2009; 53: 581-8. 